\title{
Domain structures in single crystal magnetite below the Verwey transition as observed with a low-temperature magnetic force microscope
}

\author{
Katerina Moloni ${ }^{1}$ \\ Physics Department, University of Minnesota, Minneapolis
}

\author{
Bruce M. Moskowitz \\ Department of Geology and Geophysics and Institute for Rock Magnetism, University of Minnesota, Minneapolis
}

\author{
E. Dan Dahlberg \\ Physics Department, University of Minnesota, Minneapolis
}

\begin{abstract}
The magnetic domain structures on the $\{110\}$ plane of magnetite $\left(\mathrm{Fe}_{3} \mathrm{O}_{4}\right)$ below the Verwey transition $\left(\mathrm{T}_{\mathrm{v}}=120 \mathrm{~K}\right)$ were studied using a Low-Temperature Magnetic Force Microscope (LTMFM). At 298K, domain structures consisted of arrays of $180^{\circ}, 109^{\circ}$ and $71^{\circ}$ walls, typical for magnetite with cubic anisotropy. At $77 \mathrm{~K}$ (below $\mathrm{T}_{\mathrm{v}}$ ), the cubic style patterns disappeared and transformed into uniaxial patterns consistent with the uniaxial magnetocrystalline symmetry of the low-temperature monoclinic phase of magnetite. We also observed two distinct styles of domain patterns below $T_{V}$ : (1) wide domains separated by straight $180^{\circ}$ walls along the in-plane [100] easy axis; and (2) intricate wavy walls with reverse spike domains characteristic of out-ofplane easy axes. This intimate mixture of domain styles within adjacent areas of the crystal reflects variations in the direction of the magnetic easy axes in different regions produced by caxis twinning of the crystal below $T_{v}$. The thermal dependence of planar and wavy-wall patterns show little change from $77 \mathrm{~K}$ until $110 \mathrm{~K}$, where patterns disappear. Upon cooling back to $77 \mathrm{~K}$, domain structures are different from the initial $77 \mathrm{~K}$ states, indicating that renucleation of different domain states occurs by cycling near $T_{\mathbf{v}}$.
\end{abstract}

\section{Introduction}

The Verwey transition in magnetite $\left(T_{v}=120 K\right)$ has been extensively studied since its discovery [Verwey, 1939]. It is a first order crystallographic phase transition for near stoichiometric compositions and produces rapid changes in several different physical properties upon cycling through $T_{V}$ [e.g., Kakol, 1990]. At the Verwey transition, the crystallographic structure of magnetite changes from cubic $\left(\mathrm{T}>\mathrm{T}_{\mathrm{v}}\right)$ to monoclinic $\left(\mathrm{T}<\mathrm{T}_{\mathrm{v}}\right)$ [Otsuka and Sato, 1986; Miyamota and Chikazumi, 1988]. Accompanying this crystal transition is a change in magnetocrystalline anisotropy from

${ }^{1}$ Now at Purdue University, W. Lafayette, IN

Copyright 1996 by the American Geophysical Union.

Paper number 96GL00962

0094-8534/96/96GL-00962\$05.00 cubic to uniaxial, where the magnetic easy axis in the lowtemperature phase is the monoclinic c-axis, which nearly corresponds with a particular cubic [100] direction in the hightemperature phase [e.g., Kakol. 1990]. At a temperature about $10 \mathrm{~K}$ above $T_{V}$, known as the isotropic point $\left(T_{j}\right)$, the first order magnetocrystalline anisotropy constant $\left(K_{1}\right)$ goes through zero as it changes sign and the easy directions of magnetization change their orientation from [111] above $T_{i}$ to [100] below $\mathrm{T}_{\mathrm{i}}$ [Stacey and Banerjee, 1974]. The magnetic consequences of the isotropic point and the phase transition are twofold. First, domain walls (DW) in multidomain (MD) grains will broaden as $K_{1}$ decreases near $T_{i}$ because the width of a DW is proportional to $\sqrt{ } \mathrm{A} / \mathrm{K}_{\text {eff }}$, where $A$ is the exchange constant and $K_{\text {eff }}$ is the effective anisotropy constant resulting from all anisotropy sources [e.g., Stacey and Banerjee, 1974]. As the wall width increases, the effectiveness of DW pinning by localized defects is reduced [Xu and Merrill, 1989; Moskowitz 1993] leading eventually to unpinning of walls and remagnetization. Secondly, upon passing through the phase transition at $T_{v}$, a complete reorganization of the domain structure is expected [e.g., Halgedahl and Jarrard, 1995] due to the change in magnetocrystalline anisotropy from cubic to uniaxial symmetry.

The Verwey transition is easily identified magnetically by its distinctive signatures in remanence, susceptibility, and coercivity in particles spanning the single domain $(>37 \mathrm{~nm})$ to multidomain size ranges [e.g., Nagata et al., 1965; Özdemir et al., 1993; Moskowitz et al., 1993; Halgedahl and Jarrard, 1995]. For example, a saturation remanence (SIRM) given either above or below this transition will be reduced or demagnetized upon passing through $T_{\mathbf{v}}$ in zero field. This diagnostic magnetic behavior has been used as a means for identifying the presence of this phase and, more recently, to document magnetochemical alteration in sediments and soils [e.g., Banerjee et al., 1993; Tarduno, 1995].

In this paper we report the first study of surface domain structures on the $\{110\}$ plane of single crystal magnetite below the Verwey transition using a Low-Temperature Magnetic Force Microscope (LTMFM). The LTMFM, constructed in our laboratory, can image magnetic domain structures at variable temperatures $(77 \mathrm{~K}-300 \mathrm{~K})$ with a spatial resolution of $\approx 50 \mathrm{~nm}$ under normal modes of operation. However, the weak magnetic signal associated with the 
relatively low $\mathrm{M}_{\mathrm{s}}$ of magnetite coupled with background thermal noise in our LTMFM, limit domain observations to the temperature interval between $77 \mathrm{~K}$ and $110 \mathrm{~K}$.

\section{Experimental Procedure}

Magnetic Force Microscopy (MFM) is a relatively new technique for imaging micromagnetic structures in magnetite and other magnetic materials [e.g., Williams et al., 1992; Proksch et al. 1994]. The central component of an MFM is a sharp, magnetically coated tip attached to a flexible cantilever. Our microscope employs a dynamic AC mode of operation. Images obtained with this detection mode provide a map of the force derivatives associated with the stray fields arising from magnetostatic charges produced by the divergence of the magnetization [e.g. Sarid, 1991; Proksch et al., 1994]. One should note that we can simply think of the MFM as a surface charge detector and any contrast in the images is thus due to the presence of free surface poles. The images obtained with the LTMFM contain a mixture of both topographic and magnetic information. By scanning at a sufficient height $(\approx 100 \mathrm{~nm})$ above the sample, however, the short-range topographic forces are greatly attenuated relative to the longrange magnetic ones.

The LTMFM is described in detail in Moloni [1995] and only a brief description is given here. The MFM head is placed inside a liquid-nitrogen cryostat using $\mathrm{N}_{2}$ as an exchange gas. Heating above $77 \mathrm{~K}$ is achieved with a small resistance heater inserted into the $\mathrm{Cu}$-block upon which the sample is mounted. An optical interferometry technique [Sarid, 1991] is used, with a specially designed optical fiber-cantilever holder, to monitor the deflection of the cantilever at low temperatures. However, due to both the large size of the holder apparatus and the extensive thermal path connecting the heater to the fibercantilever holder, thermal instability results in fluctuations of the fiber-cantilever distance and corresponding noise in the detection signal, for $\mathrm{T}>90 \mathrm{~K}$.

The tips used for this study are commercially available $\mathrm{Si}$ tips, coated with a thin film of CoCr. Before each set of images, the tip is magnetized in the z-direction (perpendicular to the cantilever and the sample), which coincides with the oscillation direction of the cantilever. The response of the MFM is then mostly sensitive to the variation of the $\mathrm{z}$ component of the second derivatives of stray fields along the z-direction. The high degree of sensitivity to variations in these fields allows us to systematically map out the surface magnetic structure of the sample.

$\mathrm{A} \mathrm{Fe}_{3} \mathrm{O}_{4}$ single crystal, grown along the $\langle 110\rangle$ direction, was used in our study [Wanamaker and Moskowitz, 1994]. Two disk-shaped samples approximately $1 \mathrm{~mm}$ thick were cut parallel to a $\{110\}$ plane from the original crystal and oriented using the back reflection Laue technique. Samples were mechanically polished with diamond polishing compounds followed by a final treatment using amorphous silica solution to achieve a smooth, nearly strain-free surface [Hoffmann et al., 1987]. The surface of one crystal was slightly misoriented $\left(<5^{\circ}\right)$ with respect to the $\{110\}$ plane after a second polishing, as evidenced by arrays of tree-like surface domain patterns at room temperature [Craik and Tebble, 1965]. The surface of the other sample was almost perfectly parallel to the $\{110\}$ plane as evidenced by domains bounded by $180^{\circ}, 109^{\circ}$, and $71^{\circ}$ walls associated with the presence of two in-plane $\langle 111\rangle$ easy axes [e.g., Özdemir et al., 1995].
Bulk magnetic measurements on one piece of the crystal were made at room temperature and above using a vibrating sample magnetometer and at low temperature using the Quantum Design MPMS2 and Lakeshore AC susceptometers. The saturation magnetization at $298 \mathrm{~K}$ was $92 \mathrm{Am}^{2} / \mathrm{kg}$ and the Curie temperature was $846 \mathrm{~K}$. Both measured values are typical for chemically pure magnetite. Low-temperature behavior of saturation remanence (SIRM) and AC susceptibility $(\chi)$ show abrupt change at $T=123 \mathrm{~K}$ and $125 \mathrm{~K}$ respectively, marking the location of the Verwey transition.

\section{Results}

Below $T_{v}$, the magnetic easy axis is the c-axis of the monoclinic structure, which nearly coincides with one of the three original cubic $<100\rangle$ directions. In our case for a $\{110\}$ plane, the uniaxial easy axis can be oriented along the inplane $\langle 100\rangle$ direction or oriented $45^{\circ}$ out-of-plane along the other two $<100\rangle$ directions that intersect the $\{110\}$ plane. This results in the formation of various distinct surface domain structures depending on the orientation of the easy axis with respect to the $\{110\}$ observing plane. In the MFM images presented here, the crystal is oriented such that the $\langle 100\rangle$ cubic direction runs from "top-to-bottom" in all the images.

An example of uniaxial domain patterns in magnetite in its monoclinic form at $77 \mathrm{~K}$ is shown in Fig. 1. The surface domains in this image consist of a wavy-wall structure. A small, circular, reverse polarity spike-domain is also present (Fig. 1a). These types of features are commonly observed on planes perpendicular to the easy axis of uniaxial ferromagnets when $\mu_{0} \mathrm{M}_{\mathrm{s}}{ }^{2} / 2 \mathrm{~K}_{\text {eff }}<1$ [e.g., Halgedahl, 1987]. Because the anisotropy constant for magnetite is much higher at $77 \mathrm{~K}$ than at $300 \mathrm{~K}$, this condition is easily satisfied for the monoclinic form of magnetite, where $M_{\mathrm{s}}=500 \mathrm{KA} / \mathrm{m}$ and $\mathrm{K}_{\mathrm{a}}=2.5 \times 10^{5} \mathrm{~J} / \mathrm{m}^{3}$ [Abe et al., 1976] at 77K.

In the area of the crystal imaged in Fig. 1 the domain style indicates that the easy axis is along one of the out-of-plane $<100\rangle$ directions, so that the direction of $M_{S}$ within the domains intersects the surface, producing a surface distribution of magnetic poles. The magnetic contrast associated with the wavy-wall structures in Fig. 1 confirms this interpretation.

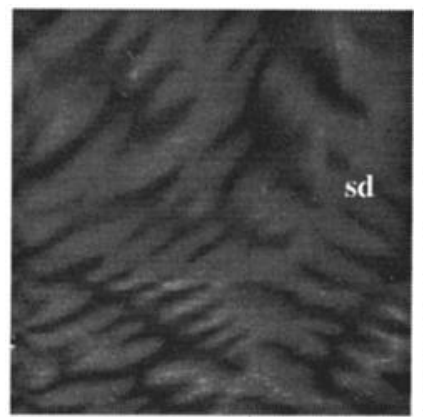

(a)

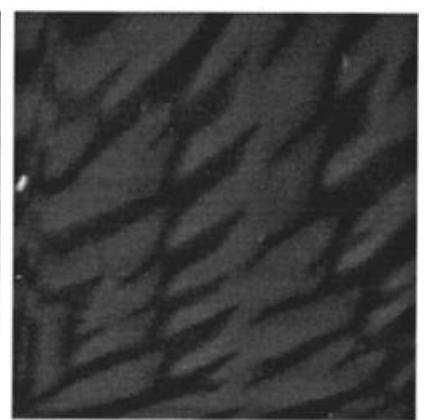

(b)
Figure 1. Two $25 \mu \mathrm{m} \times 25 \mu \mathrm{m}$ images of wavy-wall patterns at $77 \mathrm{~K}$. The contrast is due to free magnetic poles on the surface of the sample. A small spike domain (sd) may be observed in the upper right quarter of the image in (a). The image in (b) is the same area as in (a) but was taken after the sample was heated to $\mathrm{T}=111 \mathrm{~K}$ and cooled back to $77 \mathrm{~K}$. The cubic [100] direction runs from top to bottom in the image. 


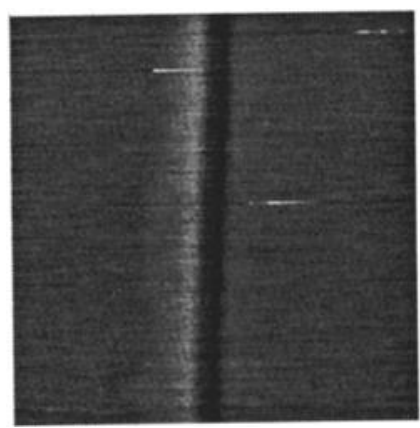

(a)

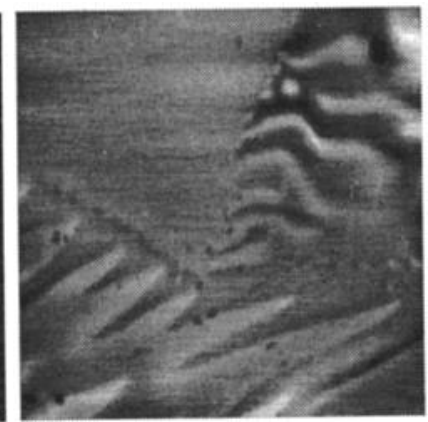

(b)
Figure 2. (a) A $4 \mu \mathrm{m} \times 4 \mu \mathrm{m}$ image of two planar domains separated by a $180^{\circ}$ Bloch wall at $77 \mathrm{~K}$. The cubic [100] direction runs from top to bottom in the image. (b) A $20 \mu \mathrm{m} \times 20 \mu \mathrm{m}$ image taken at $77 \mathrm{~K}$. The black-white contrast is due to out-of-plane magnetization, while the "empty" area in the upper left corner indicates in-plane magnetization. The cubic [100] direction runs from top to bottom in the image.

The light and dark areas result from the presence of free poles on the surface associated with oppositely magnetized domains rather than magnetic poles associated with the surface termination of walls. This indicates that the direction of $\mathbf{M}_{\mathbf{S}}$ within the domains is controlled by magnetocrystalline anisotropy, even very near the surface, rather than magnetostatic effects which would tend to rotate the domain magnetization parallel to the surface and form a completely different style of closure domains [ $X u$ and Dunlop, 1994].

In other areas of the crystals, planar domains separated by straight $180^{\circ}$ walls were observed instead (Fig. 2a). Unlike the image in Fig. 1 the magnetic contrast in Fig. $2 \mathrm{a}$ is due to the stray field distribution from the surface termination of the $180^{\circ}$ domain wall. In all cases observed, the $180^{\circ}$ walls were aligned along the original, in-plane cubic [100] direction, which coincides with the in-plane easy axis.

We also observed that both in-plane and out-of plane domain magnetizations can occur simultaneously within the same image (Fig. 2b). Here a sharp magnetic contrast is apparent only in part of the image, indicative of an out-of-plane easy axis. The "empty" space in the upper left region of Fig. 2 b indicates that the magnetization lies in-plane, but there is no $180^{\circ}$ wall within the scanning area. Such sharp changes in the direction of magnetization are mostly likely due to transformational twinning of magnetite below $T_{v}$, which allows different areas of the crystal to have different easy axis (c-axis) orientations. High resolution electron microscopy on the low-temperature phase of magnetite by Otsuka and Sato [1986] clearly show that different areas of the crystal have caxes oriented parallel to or out of the image plane. The sharpness of the magnetic boundary and the lack of any type of closure features crossing the boundaries between the in-plane and out-of-plane regions suggest that the transformational induced twinning makes the low-temperature phase of magnetite a magnetic polycrystal, with magnetically independent regions.

The thermal dependence of the domain structures between $77 \mathrm{~K}$ and $111 \mathrm{~K}$ was also studied. Fig. 3 shows a series of images corresponding to a thermal scan starting at $77 \mathrm{~K}$. Two topographical features $(\mathrm{r} 1, \mathrm{r} 2)$ serve as reference points. Initially, three planar domains separated by two $180^{\circ}$ walls are observed, consistent with an in-plane easy axis. The walls are along the [100] in-plane easy axis, which in this case runs from top to bottom in the images but is not perpendicular to any of the scanning directions, due to the way the sample was placed in the cryostat. The sample from which these images are taken has the surface slightly misoriented with respect to the $\{110\}$ plane. Thus, the domain magnetization is not exactly parallel to the $\{110\}$ plane but intersect the surface at a very small angle $\left(<5^{\circ}\right)$, creating the observed contrast in the images. Upon warming above $77 \mathrm{~K}$ the imaging area shifts towards the right and bottom of the image due to thermal expansion of the microscope head. This is the reason for not being able to observe $\mathrm{r} 2$ in Fig. 3a and 3d (taken at $77 \mathrm{~K}$ ) and the left wall in Fig. 3b. Upon warming to $99 \mathrm{~K}$ the other wall remains pinned near $\mathrm{r} 1$ until eventually disappearing at $111 \mathrm{~K}$. It is difficult to distinguish anything at this noise level, but r2 may still be seen at $\mathrm{T}=111 \mathrm{~K}$. Cooling back to $77 \mathrm{~K}$, one wall reappears close to its original position in a slightly different
77K

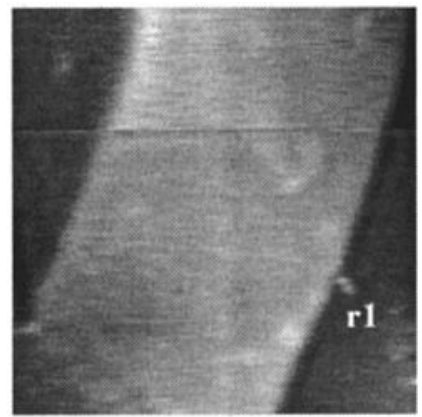

(a)
99K

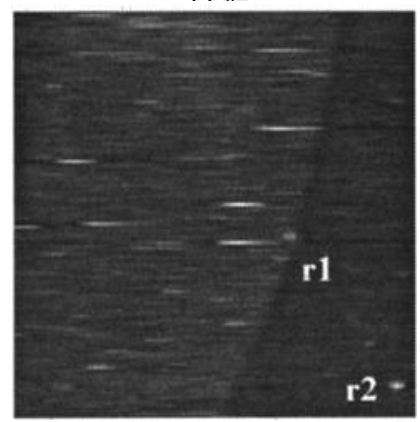

(b)
111K

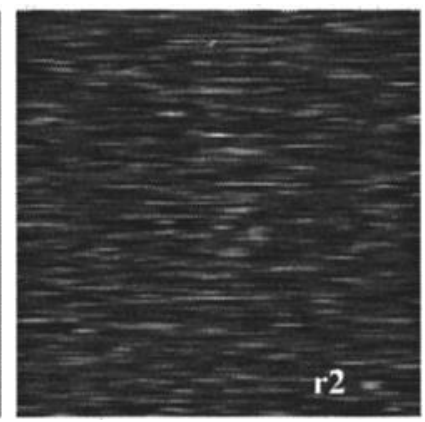

(c)
77K

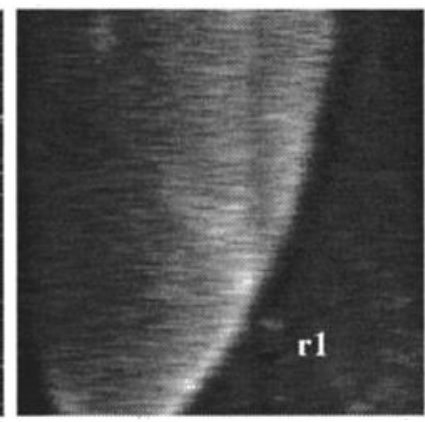

(d)

Figure 3. A series of $12 \mu \mathrm{m} \times 12 \mu \mathrm{m}$ images taken at several different temperatures during a heating and cooling cycle between $77 \mathrm{~K}$ and $111 \mathrm{~K}$. The initial domain configuration at $77 \mathrm{~K}$ is shown in (a) where three domains and two domain walls can be observed. The imaging area moves slightly to the right and bottom upon warming above 77K due to small thermal expansion of the microscope head. This is why the wall on the left is only visible in (a). The other wall remains pinned near topographical feature $\mathrm{r} 1$ and disappears at $111 \mathrm{~K}$ (c). It returns albeit in a different configuration, upon cooling back to 77K (d). Notice that topographical feature $\mathrm{r} 2$ may still be identified at the highest temperature. The apparent wall curvature is an artifact caused by the nonlinearity of the piezoscanner which is less noticeable in small scans. 
domain configuration from the initial state. Like the planar domains, the wavy-wall patterns of Fig. 1a hardly change at all by $\mathrm{T}=99 \mathrm{~K}$ until they eventually fade and disappear into the background noise by $\mathrm{T}=110 \mathrm{~K}$ (data not shown). However, cooling back to $77 \mathrm{~K}$, the structure completely changes to that shown in Fig. 1b. The changes in domain structures upon approaching $T_{v}$ are reflected in the bulk magnetization measurements that show rapid changes in SIRM beginning near $110 \mathrm{~K}$, where a reorganization of the domain structure from uniaxial to cubic patterns is expected to commence. Unfortunately, we have been unable to observe domain structures forming at temperatures above $T_{V}$ in either sample. We attribute this fact primarily to the noise associated with the cantilever-fiber distance fluctuations.

\section{Conclusions}

Domain structures at $77 \mathrm{~K}$ (below $\mathrm{T}_{\mathrm{V}}$ ) consisted of both planar domains separated by $180^{\circ}$ walls and more complicated wavywall structures. These domain patterns are similar to those predicted by classical domain theory for a material with uniaxial anisotropy and is consistent with the uniaxial magnetocrystalline symmetry of the low-temperature phase of magnetite. The two different domain styles result from inplane and out-of-plane magnetizations due to transformational twinning of magnetite below $T_{v}$, where different areas of the crystal can have different easy axis (c-axis) orientations.

Planar and wavy-wall patterns show little change from $77 \mathrm{~K}$ until $110 \mathrm{~K}$, indicating that the thermal dependence is weak. Few changes in the domain style are expected to occur below $110 \mathrm{~K}$, since the temperature dependence of the anisotropy constant ( $\mathrm{K}_{\mathrm{a}}$ in the monoclinic phase) is not a strongly varying function of temperature until very near $\mathrm{T}_{\mathbf{V}}$ [Abe et al., 1976]. At $110 \mathrm{~K}$, domain patterns disappear as the magnetic contrast is lost within the thermal background signal of the LTMFM. The fading of the domains at $110 \mathrm{~K}$ corresponds to the onset of rapid changes in SIRM of the same sample, and probably represents the initial stages of the reorganization of the domain structure from a uniaxial to a cubic type. The fact that the domain structures are different from the initial $77 \mathrm{~K}$ states after cooling back to $77 \mathrm{~K}$ from $110 \mathrm{~K}$ shows that renucleation of different domain states has occurred.

Acknowledgments. The authors would like to thank S. Foss and T. Pokhil for useful discussions. This work was supported by ONR grants N/N00014-94-I-0123 and N/N00014-89-J-1355 (EDD) and NSF grant EAR-9304520 (BMM). This is contribution 9602 of the Institute for Rock Magnetism (IRM). The IRM is supported by grants from the Keck Foundation and the NSF.

\section{References}

Abe, K., Y. Miyamota, and S. Chikazumi, Magnetocrystalline anisotropy of low temperature phase of magnetite, J. Phys. Soc. Jpn, 41, 1894$1902,1976$.

Banerjee, S.K., et. al., Separation of local signals from the regional paleomonson record of the Chinese loess plateau: a rock-magnetic approach, Geophys. Res. Lett. 20, 843-846, 1993.

Craik, D.J., and R.S. Tebble, Ferromagnetism and Ferromagnetic Domains, North-Holland Publishing Co., Amsterdam, 1965.

Halgedahl, S.L., Domain pattern observations in rock magnetism: progress and problems, Phys. Earth Planet. Int., 46, 127-163, 1987.
Halgedahl, S.L., and R.D. Jarrard, Low-temperature behavior of singledomain through multidomain magnetite, Earth Planet. Sci. Lett., 130, 127-139, 1995.

Hoffmann, V., R. Schäfer, E. Appel, A. Hubert, and H. Soffel, First domain observations with the magnetooptical Kerr effect on Tiferrites in rocks and their synthetic equivalents, $J$ Magnet. Magnet. Mat., 7I, 90-94, 1987.

Kakol, Z., Magnetic and Transport Properties of Magnetite in the Vicinity of the Verwey Transition, J. Solid State Chem., 88, 104 (1990).

Miyamota, Y., and S. Chikazumi, Crystal symmetry of magnetite in low temperature phase deduced from magnetoelectric measurements, $J$. Phys. Soc. Jpn., 57, 2040-2050, 1988.

Moloni, K., Low temperature magnetic force microscopy, $\mathrm{Ph}$. D. thesis, Un. of Minnesota, 1995.

Moskowitz, B.M., Micromagnetic study of the influence of crystal defects on coercivity in magnetite, J. Geophys. Res., 98, 18,01118,026, 1993.

Moskowitz, B.M., R.B. Frankel, and D.A. Bazylinski, Rock magnetic criteria for the detection of biogenic magnetite, Earth Planet. Sci. Lett., 120, 283-300, 1993.

Nagata, T., K. Kobayashi, and M. Fuller, Identification of magnetite and hematite in rocks by magnetic observations at low temperatures, $J$. Geophys. Res., 69, 2111-2120, 1965.

Otsuka, N. and H. Sato, Observation of the Verwey Transition in $\mathrm{Fe}_{3} \mathrm{O}_{4}$ by High-Resolution Electron Microscopy, J. Solid State Chem. 6I, 212-222, 1986.

Özdemir, Ö, D. J. Dunlop and B. M. Moskowitz, The effect of oxidation on the Verwey transition in magnetite, Geophys. Res. Lett., 20, 16711674, 1993.

Özdemir, Ö., S. Xu, and D.J. Dunlop, Closure domains in magnetite, $J$. Geophys. Res., 100, 2193-2209, 1995.

Proksch, R., S. Foss, E. D. Dahlberg, High resolution magnetic force microscopy of domain wall fine structures, IEEE Trans. Mag., 30 , 4467-4469, 1994.

Sarid, D., Scanning Force Microscopy, Oxford University Press, 1991.

Stacey, F.D., and S.K. Banerjee, The Physical Principles of Rock Magnetism, 195 pp., Elsevier, New York, 1974.

Tarduno, J.A., Superparamagnetism and reduction diagenesis in pelagic sediments: enhancement or depletion, Geophys. Res. Lett., 22, 1337 $1340,1995$.

Verwey, E.J.W., Electronic conduction of magnetite $\left(\mathrm{Fe}_{3} \mathrm{O}_{4}\right)$ and its transition point at low temperatures, Nature, 144, 327-328, 1939.

Wanamaker, B.J., and B.M. Moskowitz, Effects of nonstoichiometry on the magnetic and electrical properties of synthetic single crystal $\mathrm{Fe}_{2.4} \mathrm{Ti}_{0.6} \mathrm{O}_{4}$, Geophys. Res. Lett. $21,983-986,1994$.

Williams, W., V. Hoffmann, F. Heider, T. Göddenhenrich, and C. Heiden, Magnetic force microscopy imaging of domain walls in magnetite, Geophys. J. Int., 111, 417-423, 1992.

$\mathrm{Xu}, \mathrm{S}$., and R.T. Merrill, Microstress and microcoercivity in MD grains, J. Geophys. Res., 94, 10627-10636, 1989.

$\mathrm{Xu}, \mathrm{S}$., and D.J. Dunlop, Micromagnetic modeling of two-dimensional domain structures in magnetite, J. Geophys. Res., 99, 9035-9044, 1994.

Katerina Moloni, Physics Department, Purdue University, W. Lafayette, IN 47907-1396. (e-mail: moloni@physics.purdue.edu) Bruce M. Moskowitz, Department of Geology and Geophysics, University of Minnesota, Minneapolis, MN 55455. (e-mail: bmosk@maroon.tc.umn.edu)

E. Dan Dahlberg, Physics Department, University of Minnesota, Minneapolis, MN 55455. (e-mail: dand@physics.spa.umn.edu)

(Received January 4, 1996; revised March 11, 1996; accepted March 11, 1996.) 